\title{
Aportes de la nueva guía de tabaco: Guía basada en la evidencia del Gobiemo de la Ciudad de Buenos Aires para el tratamiento del tabaquismo
}

Contributions to the new guide of smoking: Evidence-Based Guide of the Government of the City of Buenos Aires for treatment of smoking

Gobierno de la Ciudad de Buenos Aires. Guía para el tratamiento del tabaquismo basada en la evidencia. Año 2010.

\section{Introducción}

Durante 2006 hemos resumido en dos entregas la Guía Nacional de Tratamiento de la Adicción al Tabaco del Ministerio de Salud ${ }^{1,2}$ que recomendamos leer previamente a este artículo. Durante 2010 se ha publicado la Guía para el tratamiento del tabaquismo basada en la evidencia del Gobierno de la Ciudad Autónoma de Buenos Aires ${ }^{3}$ (CABA), que tiene como objetivo actualizar conceptos y dar herramientas sencillas para utilizar en la práctica diaria en el tratamiento de la adicción al tabaco.

En relación a la metodología con la cual se llevo a cabo esta guía cabe aclarar que los autores revisaron la bibliografía disponible hasta agosto del 2009. La categorización de la fuerza de las recomendaciones que se ha establecido en la presente sigue la empleada en la Guía de Nueva Zelanda $(2007)^{4}$ y se describe en el cuadro 1.

Cuadro 1: categorización de la fuerza de las recomendaciones de la presente guía.

A: sustentada en evidencia buena (fuerte).

$\mathrm{B}$ : sustentada en evidencia suficiente (razonable) pero puede haber incertidumbre o inconsistencia mínima.

C: sustentada en opinión de expertos (publicada) solamente.

I: evidencia insuficiente.
Detallaremos a continuación los nuevos aportes de esta guía.

\section{Tratamientos Farmacológicos}

La primera línea terapéutica disponible en la actualidad para el tratamiento de la adicción al tabaco incluye a la terapia de reemplazo nicotínico, al bupropion y al varenicline; y la segunda línea, a la nortriptilina y la clonidina.

\section{Terapia de reemplazo nicotínico}

Es el primer tratamiento utilizado y ha demostrado ser altamente costo-efectivo duplicando las tasas de cesación. Son contraindicaciones relativas para el mismo ser menor de 18 años, el embarazo y la lactancia, las arritmias severas que ponen en riesgo la vida, la enfermedad cardiovascular severa aguda, la enfermedad vascular periférica y la enfermedad de Raynaud.

La tabla 1 resume las principales características de las diferentes vías de administración de esta terapia.

Tabla 1: principales características de las formas de presentación de la terapia de reemplazo nicotínico.

\begin{tabular}{|c|c|c|c|c|}
\hline & Chicles & Spray nasal & Parches & Losange \\
\hline Forma & $\begin{array}{l}\text { Resina de polacrilex que contiene } \\
\text { nicotina para absorción a nivel de la } \\
\text { mucosa oral. }\end{array}$ & $\begin{array}{l}\text { Aerosol de nicotina para absorción en } \\
\text { mucosa nasal. }\end{array}$ & $\begin{array}{l}\text { Parche adhesivo de nicotina para } \\
\text { absorción transdermica. }\end{array}$ & $\begin{array}{l}\text { Caramelo que se disuelve en la } \\
\text { boca. }\end{array}$ \\
\hline Contra-indicaciones & $\begin{array}{l}\text { Alteraciones en la mucosa oral y } \\
\text { trastornos de la masticación. }\end{array}$ & Hipersensibilidad 0 alergia nasal. & $\begin{array}{l}\text { Enfermedades de la piel que } \\
\text { impidan la colocación. }\end{array}$ & $\begin{array}{l}\text { Reacciones de hipersensibilidad } \\
\text { y enfermedades de la mucosa } \\
\text { oral. }\end{array}$ \\
\hline Efectos adversos & $\begin{array}{l}\text { Gastritis, dolor en la articulación } \\
\text { temporo-mandibular y úlceras en la } \\
\text { mucosa oral. }\end{array}$ & $\begin{array}{l}\text { Palpitaciones, irritación nasal y } \\
\text { cefalea. }\end{array}$ & $\begin{array}{l}\text { Reacciones de hipersensibilidad y } \\
\text { trastornos del sueño. }\end{array}$ & $\begin{array}{l}\text { Palpitaciones, irritación de la } \\
\text { mucosa oral, cefalea y nauseas. }\end{array}$ \\
\hline Presentación & 2 y $4 \mathrm{mg}$. & 200 pulsaciones de $50 \mathrm{mcl}$. & $7 \mathrm{mg}, 14 \mathrm{mg}$ y $21 \mathrm{mg}$. & 2 y $4 \mathrm{mg}$. \\
\hline Dosis & La dosis máxima son 24 chicles por día. & $\begin{array}{l}\text { Cada dosis de dos puffs provee } 1 \mathrm{mg} \\
\text { de nicotina, recomendándose una a } \\
\text { dos dosis por hora, con un máximo de } \\
40 \text { puffs diarios. }\end{array}$ & $\begin{array}{l}21 \mathrm{mg} \text { durante } 4 \text { semanas, } 14 \mathrm{mg} \\
\text { durante dos semanas y } 7 \mathrm{mg} \\
\text { durante dos semanas. }\end{array}$ & $\begin{array}{l}\text { La dosis máxima son } 24 \\
\text { caramelos por día. }\end{array}$ \\
\hline Duración del tratamiento & 12 semanas. & $\begin{array}{l}\text { Tres meses con descenso progresivo } \\
\text { hasta los seis meses }\end{array}$ & En general ocho semanas. & En general 12 semanas. \\
\hline $\begin{array}{l}\text { Consideraciones } \\
\text { especiales }\end{array}$ & $\begin{array}{l}\text { Se utilizan de } 402 \text { mg según el } \\
\text { paciente fume más o menos de } 20 \\
\text { cigarrillos diarios. El chicle se debe } \\
\text { masticar lentamente hasta que } \\
\text { aparezca un sabor picante, } \\
\text { colocándoselo durante } 30 \text { segundos en } \\
\text { el carrillo desde donde se absorbe, } \\
\text { repitiéndose la operación a los } 30 \\
\text { segundos. }\end{array}$ & $\begin{array}{l}\text { Es un spray nasal, el paciente debe } \\
\text { administrar un puff en cada narina } \\
\text { cuidando de esperar unos segundos } \\
\text { hasta respirar, ya que la nicotina se } \\
\text { absorbe en la mucosa nasal. }\end{array}$ & $\begin{array}{l}\text { En nuestro país solo tenemos } \\
\text { parches de } 24 \text { hs, en algunos } \\
\text { casos se pueden retirar por la } \\
\text { noche. }\end{array}$ & $\begin{array}{l}\text { Se utilizan caramelos de } 204 \\
\text { mg según el paciente fume el } \\
\text { primer cigarrillo antes o después } \\
\text { de los } 30 \text { minutos luego de } \\
\text { levantarse. Se lo coloca en la } \\
\text { boca y se lo debe dejar disolver, } \\
\text { sin masticarlo ni tragarlo. }\end{array}$ \\
\hline
\end{tabular}

Bupropion

Es un antidepresivo tricíclico que duplica la tasa de cesación, postulándose como mecanismo de acción un bloqueo de la recaptación de dopamina y noradrenalina. Se metaboliza en el hígado por el citocromo $\mathrm{P} 450$, por lo que debe ser utilizado con precaución cuando el paciente también recibe otras drogas que usan dicha vía de depuración. Son contraindicaciones para su uso el antecedente de convulsiones o enfermedades proconvulsivantes como tumores del sistema nervioso central, traumatismo de cráneo, trastornos de la alimentación, alcoholismo, anorexia y bulimia. 
Vareniclina

Es un agonista parcial de los receptores nicotínicos alfa 4 beta 2 y algunos estudios informan que triplica la tasa de cesación. Son contraindicaciones relativas para su uso la insuficiencia renal crónica (en estos pacientes se recomienda disminuir la dosis a la mitad) no habiendo experiencia en pacientes con epilepsia. También se recomienda tener precaución en pacientes con antecedentes de depresión, suicidio, ideación suicida u otra enfermedad psiquiátrica.

La tabla 2 describe las principales características de bupropion y varenicline.

Tabla 2: principales características de las formas de presentación del bupropion y la (o el?)varenicline.

\begin{tabular}{|c|c|c|}
\hline & Bupropion & Vareniclina \\
\hline Efectos adversos & $\begin{array}{l}\text { Boca seca, convulsiones (en pacientes con factores proconvulsivantes) e } \\
\text { insomnio. }\end{array}$ & $\begin{array}{l}\text { Nauseas, trastornos gástricos, sueños vívidos y pesadillas, insomnio y } \\
\text { trastornos del ánimo. }\end{array}$ \\
\hline Presentación & Comprimidos de 150 y 300 mg en envases de 30 y 60 comprimidos. & $\begin{array}{l}\text { Comprimidos de } 0,5 \text { y } 1 \mathrm{mg} \text { en envases que contienen } 11 \\
\text { comprimidos recubiertos de } 0,5 \mathrm{mg} \text { y } 14 \text { comprimidos recubiertos de } \\
1 \mathrm{mg} ; 11 \text { comprimidos recubiertos de } 0,5 \mathrm{mg} \text { y } 52 \text { comprimidos } \\
\text { recubiertos de } 1 \mathrm{mg} \text {; y } 11 \text { comprimidos recubiertos de } 0,5 \mathrm{mg} \text { y } 154 \\
\text { comprimidos recubiertos de } 1 \mathrm{mg} \text {. } \\
\text { También se presenta en envases que contienen } 28 \text { y } 56 \text { comprimidos } \\
\text { recubiertos de } 1 \mathrm{mg} \text {. }\end{array}$ \\
\hline Dosis & $\begin{array}{l}150 \text { a } 300 \text { mg diarios. Si se usan dos comprimidos diarios, iniciar el } \\
\text { tratamiento con } 150 \text { mg por día durante tres días, subiendo luego a } 300 \text { mg; } \\
\text { recomendándose comenzar una a dos semanas antes del momento de la } \\
\text { cesación. }\end{array}$ & $\begin{array}{l}0,5 \mathrm{mg} \text { por día durante tres días, } 0,5 \mathrm{mg} \text { dos veces por día desde el } \\
\text { cuarto al séptimo día, y } 1 \mathrm{mg} \text { dos veces por día hasta el fin del } \\
\text { tratamiento. En pacientes con clearance de creatinina entre } 30 \text { y } 50 \\
\mathrm{~mL} / \mathrm{min} \text { la dosis se debe reducir a } 1 \mathrm{mg} / \text { día. }\end{array}$ \\
\hline Duración del tratamiento & 8 a 12 semanas. & 12 semanas. \\
\hline Interacciones & $\begin{array}{l}\text { Principalmente con esteroides sistémicos, antidepresivos, anti psicóticos, } \\
\text { betabloqueantes y alcohol. }\end{array}$ & No presenta. \\
\hline \multirow{2}{*}{$\begin{array}{l}\text { Consideraciones } \\
\text { especiales }\end{array}$} & \multicolumn{2}{|c|}{ Precaución en pacientes con antecedentes de depresión, suicidio, ideación suicida u otra enfermedad psiquiátrica. } \\
\hline & $\begin{array}{l}\text { Tener cuidado en pacientes con condiciones pro-convulsivantes, debiendo } \\
\text { transcurrir entre una dosis y otra por lo menos ocho horas y la segunda } \\
\text { dosis no ser tomada luego de las } 17 \mathrm{~h} \text {. }\end{array}$ & $\begin{array}{l}\text { Se debe prescribir la toma con la ingesta de alimentos para reducir } \\
\text { los síntomas gástricos. }\end{array}$ \\
\hline
\end{tabular}

Fármacos de segunda línea

Se deben utilizar si hay múltiples fracasos previos o contraindicaciones para los de primera línea, estando disponible en Argentina, solo la clonidina.

Recomendaciones generales sobre el tratamiento farmacológico Es importante ofrecer tratamiento farmacológico a los pacientes motivados para dejar de fumar como parte de la intervención dado que duplica la chance de éxito (recomendación grado A).

Además, siempre que sea posible, se recomienda combinar el tratamiento farmacológico con intervenciones no farmacológicas en forma de consejería dado que esta combinación es mucho más efectiva que cualquiera de ellas en forma aislada (recomendación grado $\mathrm{A}$ ).

Hay situaciones en que el tratamiento farmacológico no es una opción inicial y debe ser evaluado con más precaución, sopesando los riesgos y los beneficios de cada paciente en particular. Ejemplos de estas situaciones son el embarazo, la lactancia, la adolescencia y cuando el consumo de cigarrillos es menor a diez por día. Estos casos particulares serán discutidos más adelante. Una vez que el médico decide prescribir un tratamiento farmacológico, para la elección del fármaco deberá tener en cuenta la condición clínica del paciente (comorbilidades y contraindicaciones de la medicación) su experiencia previa y sus preferencias y la experiencia del propio profesional en el manejo de cada una de las opciones disponibles.

Para aquellos pacientes con alta dependencia es aconsejable utilizar terapias combinadas, usualmente la terapia de reemplazo nicotínico con parches y/o chicles con bupropion (recomendación grado B).
La combinación de dos formas de administración de TRN, asociando una de acción rápida (chicles, spray o losange) con otra de acción sostenida (parches) aumenta las probabilidades de abstinencia.

\section{Situaciones especiales}

\section{Pacientes internados}

Una internación es una oportunidad para intervenir en el paciente fumador ya que existen dos razones que pueden motivar al paciente internado para dejar de fumar: una enfermedad que puede ser causada o agravada por el cigarrillo y que los hospitales sean ambientes $100 \%$ libres de humo.

Los pocos estudios con los que se cuenta tienen resultados alentadores, asociándose este tipo de intervención a un aumento de la tasa de abstinencia al alta medido al mes de seguimiento.

Es recomendación tipo B ofrecer tratamiento de cesación tabáquica utilizando bupropion y TRN durante la internación y acordar visitas de seguimiento relacionadas con la cesación, manteniendo el contacto por lo menos por el término de un mes. El Comité de expertos recomienda ofrecer tratamiento farmacológico a todos pacientes internados salvo que exista alguna contraindicación médica.

\section{Pacientes embarazadas}

El embarazo es una situación motivadora para dejar de fumar. Es muy común que en Buenos Aires las embarazadas sigan fumando, sólo disminuyendo el consumo en algunas ocasiones y recayendo luego del parto o al terminar la lactancia. Además, Ilama la atención la alta exposición al humo de segunda mano de las embarazadas y los niños. Es importante recordar que las 
mujeres que siguen fumando durante su embarazo exponen al feto no solo a la nicotina, sino también a las 4.500 sustancias toxicas presentes en el cigarrillo.

Excepto que exista alguna contraindicación medica, se recomienda brindar una intervención intensiva específica a todas las embarazadas y puérperas fumadoras (recomendación grado A).

\section{Pacientes con enfermedades psiquiátricas}

La prevalencia de tabaquismo es mayor en la población psiquiátrica que en la población general y alrededor del $50 \%$ de los pacientes que solicitan ayuda para dejar de fumar tienen alguna historia de síntomas depresivos en el pasado o antecedentes de alcoholismo o consumo de drogas de abuso.

Se debe brindar intervención breve para dejar de fumar a todos los pacientes con enfermedades psiquiátricas (recomendación grado $\mathrm{A}$ ). Siempre que sea posible, se debe ofrecer una intervención intensiva ofreciendo tratamiento farmacológico y psicosocial a pacientes con patología psiquiátrica que desean dejar de fumar (recomendación grado A).

Es recomendación del Comité de Expertos adecuar la medicación a la enfermedad psiquiátrica de base. Se recomienda que el tratamiento farmacológico lo realicen expertos en cesación junto con el personal de salud mental de cabecera del paciente, dado que el abordaje psico-social y farmacológico de estos casos puede requerir consideraciones particulares.

\section{Intervención en adolescentes (hasta 18 años)}

El tabaquismo en adolescentes en la CABA es un tema preocupante, por su alta prevalencia, (predominantemente en mujeres) por la temprana edad de inicio, por el fácil acceso a la compra de cigarrillos, y por la alta exposición al humo de tabaco en el ambiente. Por otro lado, los jóvenes se encuentran en su mayoría en estado pre contemplativo (no piensa en dejar de fumar) con respecto al deseo de dejar de fumar; y si bien pueden realizar algún intento para dejar de fumar, tienen muy elevadas tasas de recaídas. Los jóvenes en general subestiman el potencial adictivo de la nicotina. Por otro lado, se ha demostrado que raramente se identifica al adolescente fumador, a pesar de que numerosas encuestas demuestran que no tienen problema en admitir su consumo y que muchos desearían dejar de fumar. No tenemos herramientas farmacológicas al momento de tratar a un adolescente ya que ninguna de las drogas disponibles ha sido testeada en cuanto a su seguridad y efectividad en menores de 18 años. Es por esto que se considera de vital importancia poner en marcha las estrategias de control que propone el Convenio Marco de de la Organización Mundial de la Salud para el Control del Tabaco: ambientes libres de humo, aumento de los precios, regulación de la publicidad, promoción y patrocinio, y advertencias sanitarias en los empaques.

Se recomienda realizar intervenciones breves a todos los adolescentes fumadores y no fumadores, así como a los niños y a sus padres. También se recomienda informar sobre el tabaquis- mo pasivo, ya que el humo de tabaco ajeno es dañino para la salud de los niños; y ofrecer intervenciones no farmacológicas para aquellos adolescentes que desean dejar de fumar. Dada la baja evidencia de la eficacia del tratamiento farmacológico, éste no estaría recomendado excepto en casos especiales y con la colaboración de la familia, prefiriéndose en algunos casos seleccionados la TRN (recomendación grado B).

Intervención en fumadores de menos de diez cigarrillos por día Estos fumadores (asimismo los que fuman uno a cuatro por día) corren el riesgo de desarrollar enfermedades relacionadas con el consumo de tabaco, por lo que también deben ser identificados. Existen metabolizadores lentos de nicotina que a pesar de fumar menos de diez cigarrillos se mantienen con niveles de nicotina en sangre elevados. Por otro lado se recomienda tomar en cuenta la sensación de la persona sobre su dificultad para dejar de fumar más que el número de cigarrillos y en función de eso ofrecer la terapia con medicación. Se recomienda motivar y ofrecer intervenciones no farmacológicas a todos los fumadores de menos de diez cigarrillos por día (recomendación grado B).

Es recomendación del Comité de Expertos el uso de reemplazo nicotínico y considerar las otras intervenciones farmacológicas con drogas de primera línea (bupropión-vareniclina) en casos especiales.

\section{Comentario}

El tabaquismo es hoy en el mundo la principal causa de muerte evitable. Ocasiona alrededor de cinco millones de muertes al año, cifra que se duplicará en 20 años si no intervenimos con firmes políticas de control. Argentina no escapa a esta realidad ya que fuma un tercio de la población adulta y posee una de las más altas del mundo en la población femenina ${ }^{5}$. Debido a esta realidad cobra importancia capacitar en cesación tabáquica a todos los profesionales de la salud y mantener la información actualizada.

Esta guía nos ofrece una práctica actualización de los nuevos conocimientos en relación al tratamiento de la adicción al tabaco, incorporando herramientas para intervenir en poblaciones definidas como los menores de 18 años, las embarazadas y los pacientes internados.

En esta nueva guía no ha habido actualizaciones en relación a como realizar la evaluación del estatus de fumador y su motivación para dejar de fumar, ni otros sobre consejo antitabáquico; por lo que remitimos al lector a la Guía desarrollada por el Ministerio de Salud de la Nación durante 2005.

Se presenta en un formato de práctica lectura y a pesar de que no sigue los lineamientos del instrumento consensuado para la confección y evaluación de guías de práctica clínica (en inglés: Appraisal of Guidelines Research \& Evaluation Instrument o "AGREE"*) las recomendaciones que propone están respaldadas por evidencia científica.

\section{Débora Szeinman [ Servicio de Medicina Familiar y Comunitaria del Servicio de Medicina Familiar y Comunitaria del Hospital Italiano.}

debora.szeinman@hospitalitaliano.org.ar ]

\section{Referencias}

1. Ministerio de Salud y Ambiente de la Nación y Entidades Académicas, Científicas e Instituciones representativas del control del tabaco en Argentina. Coordinación técnica: Verónica Schoj y Ana Tambussi. Revisión del documento final: Mario Virgolini. Resúmen de la guía nacional de tratamiento de la adicción al tabaco: primera parte. Guía de Práctica Clínica. Evid. actual. páct. ambul. 9(3);88-90. May-Jun 2006.

2. Ministerio de Salud y Ambiente de la Nación y Entidades Académicas, Científicas e Instituciones representativas del control del tabaco en Argentina. Coordinación técnica: Verónica Schoj y Ana Tambussi. Revisión del documento final: Mario Virgolini. Resúmen de la guía nacional de tratamiento de la adicción al tabaco: primera parte. Guía de Práctica Clínica. Evid. actual. páct. ambul. 9 (4);115-120. Jul-Ago 2006.

3. Gobierno de la Ciudad de Buenos Aires. Guía para el tratamiento del tabaquismo basada en la evidencia. Año 2010.

http://estatico.buenosaires.gov.ar/areas/salud/programas/tabaco/archivos/guia_tratamiento_tabaquismo.pdf

4. Ministry of Health. 2007. New Zealand Smoking Cessation Guidelines. Wellington: Ministry of Health disponible en http://www.moh.govt.nz/moh.nst/pagesmh/6663/\$File/nz-smoking-cessation-guidelines-v2-aug07.pdf

5. Ministerio de Salud y Ambiente de la Nación, Programa Nacional de control de tabaco disponible en http://www.msal.gov.ar/htm/site_tabaco/info-prof.asp 